\title{
A Retrospective Cohort Study of Intravenous Immunoglobulin Therapy in the Acute Phase of Kawasaki Disease: The Earlier, the Better?
}

\author{
Wei Li $\mathbb{D},{ }^{1}$ Xiufang He, ${ }^{2}$ Li Zhang, ${ }^{1}$ Zhouping Wang $\mathbb{D},{ }^{1}$ Yanfei Wang, ${ }^{1}$ Huimei Lin, ${ }^{3}$ \\ Jia Yuan, ${ }^{1}$ Xiaofei Xie, ${ }^{1}$ Youzhen Qin $\left(1,{ }^{2}\right.$ and Ping Huang $\mathbb{1}^{1}$ \\ ${ }^{1}$ Department of Cardiology, Guangzhou Women and Children's Medical Center, Guangzhou Medical University, \\ Guangzhou 510120, China \\ ${ }^{2}$ Department of Pediatric Cardiovascular, The First Affiliated Hospital of Sun Yat-sen University, Guangzhou 510080, China \\ ${ }^{3}$ Department of Medical Record, Guangzhou Women and Children's Medical Center, Guangzhou Medical University, \\ Guangzhou 510120, China
}

Correspondence should be addressed to Youzhen Qin; qyouzhen@163.com and Ping Huang; huangping999@126.com

Received 24 March 2021; Revised 24 May 2021; Accepted 31 May 2021; Published 18 June 2021

Academic Editor: James C. Coons

Copyright (C) 2021 Wei Li et al. This is an open access article distributed under the Creative Commons Attribution License, which permits unrestricted use, distribution, and reproduction in any medium, provided the original work is properly cited.

Background. Although intravenous immunoglobulin (IVIG) is expected to prevent coronary artery abnormalities of Kawasaki disease (KD) in the acute phase, the timing and effectiveness of IVIG remain to be determined. The association of timing of IVIG administration in KD patients with coronary artery abnormalities is evaluated in this cohort study. Methods. We systematically studied KD patients from two participating institutions between 2015 and 2017. To reveal the effectiveness of IVIG treatment, these patients were classified into four groups regarding the time of IVIG treatment. Primary outcome was coronary artery abnormalities by echo at diagnosis and 12 months follow-up; secondary outcomes included inflammatory markers. Results. A total of 1281 patients were included in this study. The best time of IVIG treatment cut-off values in 12 months follow-up for predicting coronary artery abnormalities was days 7.5 of illness onset. According to the best time of IVIG treatment cut-off values, all patients were classified into 4 groups. Group 1 was defined as earlier IVIG treatment administration on days $\leq 4$ of the illness $(n=77)$. Group 2 was defined with days 5-7 $(n=817)$, group 3 with days 8-10 $(n=249)$, group 4 with days $>10(n=138)$. A greater proportion of IVIG-resistant KD patients were group 4 than the other three groups, and there were significant differences $(p<0.05)$. The incidence of coronary artery lesions (CALs) and coronary artery aneurysms (CAAs) in group 3 and group 4 was higher than that in group $1(p<0.05)$ and group $2(p<0.05)$ during a 12 -month follow-up. Additionally, the incidence of CALs in group 1 was higher than that in group 2 but without statistical significance $(p>0.05)$. The OR was significantly higher for those who started IVIG administration more than 7 days from the onset was positively associated with the occurrence of CALs (OR, 5.3; 95\% CI, 2.0-13.9) and CAAs (OR, 13.5; 95\% CI, 2.9-14.1) 12 months after initial onset. Multivariate regression revealed that the timing of IVIG treatment and IVIG-resistance was independent risk factors of CALs. Conclusions. IVIG treatment less than 7 days after illness onset are found to be sufficient for preventing developing coronary artery abnormalities in KD patients. Earlier IVIG treatment administration within 4 days may not increase the higher incidence of coronary artery abnormalities and IVIG resistance (Chinese Clinical Trial Registry:ChiCTR1800015800).

\section{Introduction}

It has been more than 50 years since Tomisaku Kawasaki reported the first case of mucocutaneous lymph node syndrome in Japanese children $[1,2]$. Kawasaki disease (KD) occurs mainly in children, which surpasses acute rheumatic fever as a leading cause of acquired heart disease [3]. Nevertheless, KD has become a leading cause of cardiac morbidity in the world.

Intravenous immunoglobulin (IVIG) therapy can remarkably reduce the high chance of causing coronary 
artery abnormalities by $20-25 \%$ to less than 5\% [4], which represents as great progress in $\mathrm{KD}$. The definitive trial by Newburger et al. [5] showed that a single $2 \mathrm{~g} / \mathrm{kg}$ dosage of IVIG is more effective at reducing the risks of developing aneurysms than the same total dose administered once per day for 4 days in 1991. The treatment of KD with IVIG proves to be one of the most cost-effective therapeutic interventions in pediatrics nowadays. Previous studies showed that high-dose IVIG is an important treatment within 10 days of illness onset and has been demonstrated effective in dramatically reducing the risk of cardiac complications [57]. However, debates about the risk for IVIG unresponsiveness and coronary artery lesions (CALs) in association with the earlier timing of IVIG administration of disease onset still remain [8]. On the other hand, these big cohort studies of the effects of IVIG on the cardiac complications of KD were still rare. So the purpose of this study was to investigate the timing of IVIG administration associated with the development of coronary artery abnormalities 12 months after the onset.

In this study, we systematically investigated $\mathrm{KD}$ patients from two institutions. By classifying them into different groups regarding the IVIG treatment time, we examined this hypothesis of IVIG therapy in the acute phase of KD: the earlier, the better.

\section{Methods}

2.1. Enrollment of Patients. After the present study was reviewed and approved by the Scientific Research Ethics Committee at Guangzhou Women and Children's Medical Center and the First Affiliated Hospital of Sun Yat-sen University (NO.2018032608565814), we conducted a retrospective case-control study admitted to 2 hospitals and diagnosed with KD. And we also obtained written informed consent from the parents or guardians of all the participating children.

The medical records of KD who were admitted to the two hospitals were reviewed to determine who met the criteria for inclusion in the study between January 1, 2015, and December 31,2017. The inclusion criteria for study subjects were as follows: (1) patients were $\leq 18$ years old and only complete and incomplete $\mathrm{KD}$ cases in accordance with the existing guidelines were reviewed; (2) patients of initial onset of the disease; (3) patients received IVIG treatment $(2 \mathrm{~g} / \mathrm{kg}$ per day) during the acute phase of illness and maintained follow-up for at least 12 months; (4) patients had serious infection, allergy, and collagen disease. Their demographic, clinical, and laboratory data echocardiography results and outcomes were extracted from the medical records. Patients were diagnosed with $\mathrm{KD}$ by two experienced pediatricians using the recommended universal KD criteria proposed by the American Heart Association [3]. Classic KD was diagnosed in any children with a fever that lasts longer than five days and manifested four of the following five criteria: (i) diffuse mucosal inflammation with strawberry tongue and fissured lips, (ii) bilateral nonpurulent conjunctivitis, (iii) changes of the peripheral extremities, (iv) polymorphous exanthema, and (v) unilateral cervical lymphadenopathy. Patients were categorized as having complete and incomplete
$\mathrm{KD}$. Incomplete $\mathrm{KD}$ was defined as in a child with prolonged unexplained fever, fewer than 4 of the principal clinical findings, and compatible laboratory or echocardiographic findings [9]. Patients with $\mathrm{KD}$ develop a recrudescent or persistent fever at least 36 hours after the end of their IVIG infusion and are termed as IVIG-resistant KD. The treatment protocol [10] included high doses of IVIG $(2 \mathrm{~g} / \mathrm{kg})$ as a single infusion over 8 to $12 \mathrm{~h}$ along with aspirin $(30-50 \mathrm{mg} / \mathrm{kg}$ per day) during the acute phase followed by $3-5 \mathrm{mg} / \mathrm{kg}$ per day for 6-8 weeks or until resolution of coronary artery abnormalities. Combined antiplatelet and anticoagulation therapy were recommended for patients with giant aneurysms.

Using the electronic medical records databases, we collected the following variables in our study: age, gender, the day of illness onset, the day of initial IVIG treatment, laboratory data, and echocardiography results. Laboratory data including white blood cell (WBC) count, neutrophils, and C-reactive protein (CRP) before and after IVIG infusion were collected for analysis. Peripheral blood was tested before IVIG treatment and after completing the initial IVIG treatment (within 3 days). We use echocardiography in the evaluation and follow-up of coronary artery abnormalities in patients. Echocardiography was performed at enrollment and 1 month, 3 months, 6 months, and 12 months after the initial onset. Echocardiography was performed and supervised by an experienced pediatric cardiographer and with appropriate transducers. Echocardiographic evaluation of coronary artery in KD patients included imaging on the left main coronary artery, anterior descending, left circumflex, right coronary artery, and posterior descending coronary arteries. We used the definition of CALs:(1)a $z$-score $\geq 2$, (2) internal diameter of a segment 1.5 times that of an adjacent segment (saccular or fusiform aneurysm), and (3) irregular surface and/or perivascular brightness of the coronary wall. Coronary artery aneurysms (CAAs) were classified as $\mathrm{Z}$ scores in this study $[4,11]$ : small aneurysm: $\geq 2.5$ to $<5$; medium aneurysm: $\geq 5$ to $<10$, and absolute dimension $<8$ $\mathrm{mm}$; giant aneurysm: $\geq 10$, or absolute dimension $\geq 8 \mathrm{~mm}$.

2.2. Statistical Analysis. The primary outcome variables for this study were the presence or absence of coronary artery abnormalities in the acute phase and 12 months follow-up, the duration of fever, the day of IVIG administered in the acute phase of $\mathrm{KD}$, and the number and proportion of IVIG resistance. In this descriptive study, means/medians and proportions were used to describe the characteristics of the study population. The laboratory parameters before and after the initial IVIG were tested using one-way ANOVA. The proportions of Coronary artery abnormalities between the groups were compared by the $\chi^{2}$ test with the Yates correction or Fisher's exact test. The tests used for statistical analysis were two tailed. Data are presented as box plots in 12 months follow-up of coronary artery abnormalities. The analysis of the time of IVIG treatment for the diagnosis of coronary artery abnormalities was assessed by the area under the curve (AUC), sensitivity, and specificity at the optimal cut point according to the Youden index. Multivariate regression analysis after adjusting for gender, age, duration of fever, timing of initial IVIG treatment, IVIG resistance, and 
laboratory parameters were used to calculate the odds ratio with a 95\% confidence interval for CALs and CAAs. All tests were two sided, and $p<0.05$ was considered significant. All statistical tests were performed with SPSS 21.0 for Windows (SPSS, Chicago, IL, USA).

\section{Results}

3.1. Demographic Data and Characteristics of Patient Population. Of 1328 patients diagnosed with acute KD in the study time frame at 2 hospitals, 1281 patients met the inclusion criteria were included in the study. Among the included patients, all patients received IVIG treatment (IVIG $2 \mathrm{~g} / \mathrm{kg}$ for per day) once the KD diagnosis was made. The patients included in our analysis are described in Table 1. In this cohort, 816 patients (56.9\%) were male, the median age was 12 months (interquartile range, 11-36 months), and $141(11 \%)$ of 1281 patients had IVIG resistant KD and received IVIG retreatment or additional anti-inflammatory treatments for $\mathrm{KD}$.

Figure 1 shows the days of illness with IVIG treatment versus a diagnosis of coronary artery abnormalities in 12 months follow-up yielded an AUC of 0.80 (95\% CI, 0.76 to $0.84, p<0.001)$. The best time of IVIG treatment cut-off values in 12 months follow-up for predicting coronary artery abnormalities was days 7.5 of illness. This cut-off value during 12 months follow-up predicted coronary artery abnormalities with sensitivity, specificity, positive predictive value, negative predictive value, and diagnostic accuracy of $88 \%, 59 \%, 79 \%, 71 \%$, and $80 \%$, respectively. According to the best time of IVIG treatment cut-off values, all patients were classified into 4 groups in this study. Group 1 was defined as earlier IVIG treatment administration on days $\leq 4$ of the illness $(n=77,6.0 \%)$. Group 2 was defined with days 5-7 $(n=817,63.8 \%)$. Group 3 with days 8-10 $(n=249,19.4 \%)$. Group 4 with days $>10(n=138,10.8 \%)$.

As shown in Table 2, there were no significant differences in age $\leq 1$ year and gender among the 4 groups. Observed higher risk of IVIG resistance was positively associated with later IVIG treatment $>10$ days of illness. There were a greater proportion of IVIG-resistant KD patients in group 4 than the other three groups and they were significant differences $(p<0.05)$.

Laboratory baseline data analysis shows markers of inflammation, such as WBC count, \% neutrophils, and CRP in Table 3. CRP and \% neutrophils were higher in group 1 and group 2 than the other two groups, and there were significant differences among the four groups before or after IVIG treatment $(p<0.05)$, suggesting inflammatory reaction is severe in $\mathrm{KD}$ of group 1 and group 2. On the other hand, no difference in the WBC count was observed in the four groups.

Table 4 shows the actual numbers and the proportion of coronary artery abnormalities of patients and the OR and 95\% confidence intervals for each variable in relation to the development of CALs and CAAs, calculated by logistic analysis. The follow-up data indicated that 97\% (1242/1281) of CALs returned to normal in terms of diameter as assessed by echocardiography in the 12 months after the illness. All of these patients had no death in the acute phase and follow-up. Furthermore, the incidence of CALs and CAAs in group 1, group 2, group 3, and group 4 at the acute phase was $14.1,19.5,35.3$, and $58.7 \% ; 2.6,3.5,8.4$, and $30.4 \%$, respectively. During 12 months follow-up, a significantly increased incidence of CALs and CAAs was observed in group 3 and group 4 compared with group 1 and group 2 . The higher incidence of CALs and CAAs in group 3 and group 4 than the other two groups and significantly different in statistical analysis during 12 months follow-up $(p<0.05)$. The incidence of CALs in group 1 was higher than that in group 2 but without statistical significance $(p>0.05)$. Multivariate logistic regression analysis showed that receipt of IVIG >days 7 of illness at one month was positively associated with the occurrence of CALs (OR, 2.1; 95\% CI, 1.43.0) and CAAs (OR, 3.9; 95\% CI, 1.9-8.2). There was a significant difference in the higher proportion of CALs for those who started IVIG administration more than 7 days from the onset was positively associated with the occurrence of CALs $(\mathrm{OR}=5.3 ; 95 \% \mathrm{CI}=2.0-13.9, p=0.001)$ and CAAs $(\mathrm{OR}=13.5 ; 95 \% \mathrm{CI}=2.9-14.1, p<0.001) 12$ months after initial onset. Multivariate regression also revealed that the timing of IVIG treatment and IVIG resistance were independent risk factors of CALs. These results also showed a significant positive correlation between the risk of coronary artery abnormalities and the time of IVIG treatment $>$ days 7 . The number and proportions of GCAAs among the four groups were low and not compared in this study.

\section{Discussion}

$\mathrm{KD}$ has become a leading cause of cardiac morbidity in children in many countries nowadays and the incidence of KD in children has increased over time, especially in Asia [12, 13]. $\mathrm{KD}$ is an acute, self-limited vasculitis of small and mediumsized arteries that leads to CALs in approximately $25 \%$ of untreated patients $[14,15]$. This breakthrough was largely based on the published report by Furusho et al. [16] about the unrelated discovery of intravenous immunoglobulin as the treatment for idiopathic thrombocytopenic purpura. Based on their initial finding, IVIG appeared to decrease the incidence of coronary artery aneurysm in patients with KD. Furusho et al. [5] published a controlled study and found that a single infusion of $2,000 \mathrm{mg} / \mathrm{kg}$ per day was more effective than a regimen of $400 \mathrm{mg} / \mathrm{kg}$ per day given for 5 days. Afterwards, a high dose of IVIG has become the cornerstone of the management of acute $\mathrm{KD}$ for decades. Timely treatment with IVIG has decreased this risk to $3 \%$ to $5 \%$ [17]. While IVIG therapy is expensive, it is relatively safe, and the benefit typically substantially outweighs the risks [18]. IVIG is a biological product of pooled donor plasma. Adverse effects vary among products. Coombs-positive hemolytic anemia and self-resolving aseptic meningitis have been reported but are rare $[19,20]$. In this work, we enrolled $1281 \mathrm{KD}$ patients from the two institutions to delineate the risk for the occurrence of CALs association with the timing of IVIG treatment.

Nowadays, it is known that KD is characterized by systemic inflammation in all the medium-sized arteries during 
TABLE 1: Baseline characteristics of 1281 Kawasaki disease patients.

\begin{tabular}{lc}
\hline Variables & No. (\%) \\
\hline No. of patients & 1281 \\
Age median (IQR), months & $12(11-36)$ \\
Age $\leq 1$ y, no. (\%) & $717(56.0)$ \\
Gender (male : female) & $1.75: 1(816: 465)$ \\
Days of first hospital visit (d) & $6(5-7)$ \\
Illness days of initial IVIG (d) & $6(5-8)$ \\
Total hospital stay (d) & $3(2-4)$ \\
IVIG-resistant KD, no. (\%) & $141(11.0)$ \\
\hline
\end{tabular}

Expressed as the median (interquartile range: IQR), or $n \%$. IVIG: intravenous immunoglobulin.

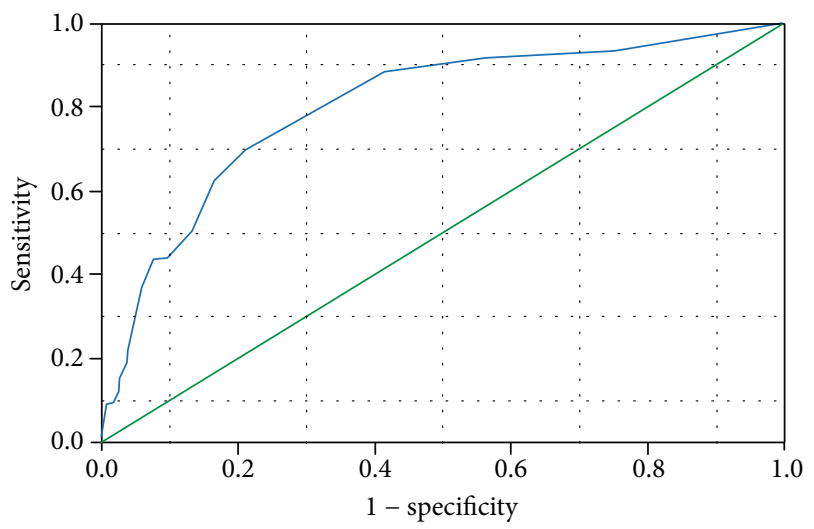

Figure 1: The time of IVIG treatment and the patients with coronary artery abnormalities due to Kawasaki disease in 12 months follow-up. Shown are receiver-operating-characteristic (ROC) curves for days of illness with IVIG treatment and coronary artery abnormalities in 12 months follow-up with $\mathrm{KD}$ cohorts. The best days of IVIG treatment cut-off values for predicting coronary artery abnormalities were 7.5 days. The area under the curve (AUC) was 0.80 (95\% CI, 0.76 to 0.84 ).

the acute febrile phase, leading to associated main clinical findings $[15,21]$. It is reported that necrotizing arteritis occurred within 2 weeks after fever onset $[15,22]$. It is only self-limited process and progressively destroys the arterial wall into the adventitia, causing aneurysms [23]. The present study is compatible with the idea that the decreased risk of cardiac complications among patients with $\mathrm{KD}$ who receive earlier IVIG treatment is due primarily to higher initial disease severity. So we attempted to observe the association between earlier treatment and cardiac complications. Numerous results suggested that IVIG treatment need not be delayed in patients diagnosed with $\mathrm{KD}$. As opposed to delaying treatment for patients with $\mathrm{KD}$ who are diagnosed earlier, the literature suggests that more aggressive treatment of patients with high initial disease severity may help protect against cardiac complications [7]. Our study observed lower risks of cardiac complications and proportions of IVIGresistance among patients treated with IVIG as early as possible. IVIG treatment $>10$ days after illness onset was consid- ered to be prone to develop IVIG resistance and high risks of cardiac complications.

As in previous reports $[24,25]$, we recorded a higher risk for development to CALs if IVIG was administrated after 10 days of disease onset. A significant beneficial effect of IVIG administration prior to 10 days of fever was also identified in randomized clinical trials [26]. Because IVIG is more likely to be able to interrupt vascular inflammation in the acute stage of illness. Additionally, some studies showed none of the IVIG responder children required readministration of IVIG to decrease the risk for development of CA pathology $[11,19,20]$. Children with KD are still at risk for coronary artery abnormalities even after IVIG administration. A risk of $8 \%$ has been reported for CALs irrespective of the administration of IVIG within 8 or after 10 days of fever onset [13]. Although IVIG treatment within 10 days was recognized as an evidence-based standard, the best time for IVIG treatment with respect to the fever onset is still debatable. Our study showed that 7 days of fever were identified as an appropriate cut-off point for IVIG administration. It is also showed that patients treated within day 7 of illness onset may develop CALs and CAAs significantly less frequently as compared to those who are treated on days $\geq 8$. We found the low-risk level for occurrence or almost complete resolution of CA abnormalities in children with $\mathrm{KD}$ with respect to the time of IVIG administration within 7 days of fever onset. Therefore, late IVIG is less effective for preventing CALs. Patients whose symptoms do not meet the classic KD criteria are problematic, and the diagnosis is frequently delayed. However, it is difficult to determine the effectiveness of IVIG after 7 days based only on coronary outcomes.

The risk factors of IVIG resistance as well as CALs and coronary aneurysms have been extensively studied [10, 27]. About one-third of patients with KD rapidly develop symptoms and are diagnosed within day 4 of illness in Japan [28, 29]. They are recognized as high risk of IVIG resistance and coronary artery abnormalities [30]. The American Heart Association scientific statement to KD treatment recommend [4] that IVIG should be administered to children as early as the diagnosis, even presenting after the 10th day of illness if they have either persistent fever without another explanation or aneurysms and ongoing systemic inflammation. It is reported that a lower rate of IVIG treatment resistance within day 5 suggested the possibility that early-administered IVIG is more likely to subside inflammation effect [31]. Similarly, in our study, the results showed IVIG treatment within day 4 of illness was reported to be not associated with increasing IVIG resistance and risk of developing coronary artery abnormalities. Some studies speculated that early IVIG treatment might have resulted in an earlier effect in immunoglobulins levels change during the acute phase of KD [32-34]. Similarly, some research investigated the precise pharmacokinetics of IVIG as well as the influence of immunological changes during the acute phase of $\mathrm{KD}$ on treatment response $[23,33,35-39]$. The optimal timing of the initial IVIG in these patients has long been discussed to improve their outcomes [17]. In our study, the proportion of IVIG resistance was $13.0 \%$ in group 1 . In line with other results, approximately $10 \%$ of patients are resistant to IVIG $[40,41]$, 
TABLE 2: The characteristics in 1281 patients in 4 groups with Kawasaki disease.

\begin{tabular}{|c|c|c|c|c|c|}
\hline Patient characteristic & Group $1(n=77)$ & Group $2(n=817)$ & Group $3(n=249)$ & Group $4(n=138)$ & $p$ value \\
\hline Age (months) & $12(7-24)$ & $12(12-36)$ & $12(11.5-36)$ & $12(8-24)$ & \multirow{2}{*}{0.009} \\
\hline Means \pm SD & $17.8 \pm 15.7$ & $23.2 \pm 19.7$ & $26.4 \pm 23.4$ & $22.3 \pm 22.0$ & \\
\hline Age $\leq 1$ yr. & $50(64.9)$ & $450(55.1)$ & $133(53.4)$ & $84(60.9)$ & 0.189 \\
\hline Gender (male: female) & $1.4: 1$ & $1.7: 1$ & $1.8: 1$ & $2.2: 1$ & 0.464 \\
\hline Days of first hospital visit (d) & $4(3-4)$ & $5(5-6)$ & $8(7-9)$ & $13(11-15)$ & - \\
\hline Illness days of initial IVIG (d) & $4(4-4)$ & $6(5-7)$ & $9(8-9)$ & $13.5(12-16)$ & - \\
\hline Total hospital stay $(\mathrm{d})$ & $3(2-5)$ & $2(2-3)$ & $2(2-4)$ & $4(2-7)$ & $<0.001$ \\
\hline IVIG-resistant KD cases & $10(13.0)$ & $79(9.7)$ & $27(10.8)$ & $25(18.1)$ & 0.03 \\
\hline
\end{tabular}

Data are presented as median (interquartile range) or $n$ (\%). IVIG: intravenous immunoglobulin. KD: Kawasaki disease.

TABLE 3: The laboratory parameters in 1281 patients in 4 groups with Kawasaki disease.

\begin{tabular}{|c|c|c|c|c|c|c|}
\hline & Laboratory parameter & Group $1(n=77)$ & Group $2(n=817)$ & Group $3(n=249)$ & Group $4(n=138)$ & $p$ value \\
\hline \multirow{3}{*}{ Baseline data } & WBC count $\left(10^{3} / \mathrm{mm}^{3}\right)$ & $15.5 \pm 6.1$ & $15.9 \pm 5.5$ & $16.2 \pm 6.6$ & $15.9 \pm 7.3$ & 0.764 \\
\hline & Neutrophils (\%) & $62.0 \pm 14.7$ & $61.7 \pm 14.6$ & $60.6 \pm 13.9$ & $55.2 \pm 14.8$ & $<0.001$ \\
\hline & CRP (mg/L) & $82.7 \pm 55.2$ & $89.4 \pm 60.7$ & $77.9 \pm 57.5$ & $67.1 \pm 56.3$ & $<0.001$ \\
\hline \multirow{3}{*}{ Data after treatment } & WBC count $\left(10^{3} / \mathrm{mm}^{3}\right)$ & $11.0 \pm 4.4$ & $9.97 \pm 4.24$ & $9.7 \pm 4.6$ & $9.6 \pm 4.2$ & 0.095 \\
\hline & Neutrophils (\%) & $37.5 \pm 15.3$ & $38.5 \pm 15.3$ & $41.1 \pm 16.0$ & $36.8 \pm 16.6$ & 0.039 \\
\hline & CRP (mg/L) & $43.1 \pm 47.1$ & $51.9 \pm 52.6$ & $36.6 \pm 40.3$ & $29.5 \pm 40.1$ & $<0.001$ \\
\hline
\end{tabular}

Expressed as means \pm SD. CRP: C-reactive protein; WBC: white blood cell.

TABLE 4: Coronary artery abnormalities for patients with Kawasaki disease in 4 groups during 12 months follow-up.

\begin{tabular}{|c|c|c|c|c|c|c|}
\hline & Cardiac complications & Acute phase & 1 month & 3 months & 6 months & 12 months \\
\hline \multirow{3}{*}{ Group $1(n=77)$} & CALs & $11(14.3)$ & $9(11.7)$ & $6(7.8)$ & $2(2.6)$ & $1(1.3)$ \\
\hline & CAAs & $2(2.6)$ & $2(2.6)$ & $1(1.3)$ & $1(1.3)$ & - \\
\hline & GCAAs & $1(1.3)$ & $1(1.3)$ & $1(1.3)$ & - & - \\
\hline \multirow{3}{*}{ Group $2(n=817)$} & CALs & $159(19.5)$ & $90(11.0)$ & $31(3.8)$ & $10(1.2)$ & $7(0.9)$ \\
\hline & CAAs & $29(3.5)$ & $14(1.7)$ & $6(0.7)$ & $5(0.6)$ & $2(0.2)$ \\
\hline & GCAAs & $3(0.4)$ & $2(0.2)$ & $2(0.2)$ & $1(0.1)$ & - \\
\hline \multirow{3}{*}{ Group $3(n=249)$} & CALs & $88(35.3)$ & $51(20.5)$ & $23(9.2)$ & $15(6.0)$ & $11(4.4)$ \\
\hline & CAAs & $22(8.8)$ & $16(6.4)$ & $13(5.2)$ & $10(4.0)$ & $8(3.2)$ \\
\hline & GCAAs & $2(0.8)$ & $2(0.8)$ & $3(1.2)$ & $3(1.2)$ & $3(1.2)$ \\
\hline \multirow{3}{*}{ Group $4(n=138)$} & CALs & $81(58.7)$ & $56(40.6)$ & $38(27.5)$ & $29(21.0)$ & $20(14.5)$ \\
\hline & CAAs & $42(30.4)$ & $30(21.7)$ & $21(15.2)$ & $20(14.5)$ & $13(9.4)$ \\
\hline & GCAAs & $6(4.3)$ & $8(5.8)$ & $8(5.8)$ & $5(3.6)$ & $5(3.6)$ \\
\hline \multirow{7}{*}{$p$ value } & Group 1 vs. 2 & 0.269 & 0.857 & 0.087 & 0.277 & 0.515 \\
\hline & CALs & $<0.001$ & $<0.001$ & 0.001 & $<0.001$ & 0.001 \\
\hline & Group 2 vs. 3 & OR 2.3 & OR 2.1 & OR 2.6 & OR 5.2 & OR 5.3 \\
\hline & & 95\% CI 1.7-3.1 & 95\% CI 1.4-3.0 & 95\% CI 1.5-4.5 & 95\% CI 2.3-11.7 & $95 \%$ CI 2.0-13.9 \\
\hline & Group 1 vs. 2 & 0.491 & 0.407 & 0.469 & 0.418 & - \\
\hline & & 0.001 & $<0.001$ & $<0.001$ & $<0.001$ & $<0.001$ \\
\hline & Group 2 vs. 3 & $\begin{array}{c}\text { OR } 2.7 \\
95 \% \text { CI } 1.5-4.7\end{array}$ & $\begin{array}{c}\text { OR } 3.9 \\
95 \% \text { CI } 1.9-8.2\end{array}$ & $\begin{array}{c}\text { OR } 7.4 \\
95 \% \text { CI } 2.8-19.8\end{array}$ & $\begin{array}{c}\text { OR } 6.8 \\
95 \% \text { CI } 2.3-20.1\end{array}$ & $\begin{array}{c}\text { OR } 13.5 \\
95 \% \text { CI } 2.9-14.1\end{array}$ \\
\hline
\end{tabular}

Expressed as $n$ (\%). CALs: coronary artery lesions; CAAs: coronary artery aneurysms; GCAAs: giant coronary artery aneurysms. OR: odds ratio. 
although our results do not provide a definitive answer to the question of when to administer the initial IVIG in earlydiagnosed KD. Patients after early IVIG treatment would be preferable to closely monitored for resistance. There have been no clinical trials comparing the effectiveness of IVIG with different days of illness onset. At the end of the midterm follow-up, no fatal case occurred. Additional medical therapies including antiplatelet therapy in patients with $\mathrm{KD}$ are administered to reduce the prevalence of CALs, reduce systemic inflammation, and prevent coronary thrombosis [42]. If CAAs are already present, antiplatelet and anticoagulation therapy is combined to reduce the risk of adverse cardiovascular events and to prevent coronary thrombosis $[43,44]$.

This was a retrospective study performed by analyzing medical records and clinic visits. Enrollment of KD patients was based on standard IVIG treatment and midterm follow-up, inevitably introducing selection bias. Because of the limitations of this study, future studies are needed.

\section{Conclusions}

IVIG treatment less than 7 days of illness onset is found to be sufficient for preventing developing coronary artery abnormalities in KD patients. Earlier IVIG treatment administration within days 4 may not increase the higher incidence of coronary artery abnormalities and IVIG resistance.

\section{Data Availability}

All data relevant to the study are included in the article.

\section{Conflicts of Interest}

The authors have declared that no conflict of interest exists.

\section{Authors' Contributions}

Wei Li and Xiufang He contributed equally to this paper. YZQ and HP conceived and designed the study. ZPW, YFW, JY, HML, ZL and XFX recruited patients and collected data. WL and XFH analyzed the data. WL drafted the manuscript and all authors edited the manuscript.

\section{Acknowledgments}

We would like to thank the patients and volunteers for their participation. This study was supported by the Guangdong Medical Research Foundation (grant no. A2018018), Guangzhou Health Science and Technology Project (grant nos. 20191A011032 and 20191A011025), and the fund from Guangzhou Institute of Pediatrics/Guangzhou Women and Children's Medical Center (grant nos. YIP-2018-027, IP2019-018, and IP-2019-020).

\section{References}

[1] T. Kawasaki, "Acute febrile mucocutaneous syndrome with lymphoid involvement with specific desquamation of the fingers and toes in children," Arerugī vol. 16, no. 3, pp. 178222, 1967.
[2] T. Kawasaki, F. Kosaki, S. Okawa, I. Shigematsu, and H. Yanagawa, "A new infantile acute febrile mucocutaneous lymph node syndrome (MLNS) prevailing in Japan," Pediatrics, vol. 54, no. 3, pp. 271-276, 1974.

[3] J. W. Newburger, M. Takahashi, M. A. Gerber et al., "Diagnosis, treatment, and long-term management of Kawasaki disease: a statement for health professionals from the committee on rheumatic fever, endocarditis, and Kawasaki disease, council on cardiovascular disease in the Young, American Heart Association," Pediatrics, vol. 114, no. 6, pp. 1708-1733, 2004.

[4] B. W. McCrindle, A. H. Rowley, J. W. Newburger et al., "Diagnosis, treatment, and long-term management of Kawasaki disease: a scientific statement for health professionals from the American Heart Association," Circulation, vol. 135, no. 17, pp. e927-e999, 2017.

[5] K. Furusho, H. Nakano, K. Shinomiya et al., "High-dose intravenous gammaglobulin for Kawasaki disease," The Lancet, vol. 2, no. 8411, pp. 1055-1058, 1984.

[6] J. W. Newburger, M. Takahashi, J. C. Burns et al., "The treatment of Kawasaki syndrome with intravenous gamma globulin," The New England Journal of Medicine, vol. 315, no. 6, pp. 341-347, 1986.

[7] T. Zhang, H. Yanagawa, I. Oki et al., "Factors related to cardiac sequelae of Kawasaki disease," European Journal of Pediatrics, vol. 158, no. 9, pp. 694-697, 1999.

[8] T. Kobayashi, Y. Inoue, K. Takeuchi et al., "Prediction of intravenous immunoglobulin unresponsiveness in patients with Kawasaki disease," Circulation, vol. 113, no. 22, pp. 26062612, 2006.

[9] J. W. Newburger, "Kawasaki disease: medical therapies," Congenital Heart Disease, vol. 12, no. 5, pp. 641-643, 2017.

[10] B. W. McCrindle and E. S. Selamet Tierney, "Acute treatment for Kawasaki disease: challenges for current and future therapies," The Journal of Pediatrics, vol. 184, pp. 7-10, 2017.

[11] F. Dallaire and N. Dahdah, "New equations and a critical appraisal of coronary artery Z scores in healthy children," Journal of the American Society of Echocardiography, vol. 24, no. 1, pp. 60-74, 2011.

[12] R. Uehara and E. D. Belay, "Epidemiology of Kawasaki disease in Asia, Europe, and the United States," Journal of Epidemiology, vol. 22, no. 2, pp. 79-85, 2012.

[13] J. J. Chen, X. J. Ma, F. Liu et al., "Epidemiologic features of Kawasaki disease in Shanghai from 2008 through 2012," The Pediatric Infectious Disease Journal, vol. 35, no. 1, pp. 7-12, 2016.

[14] J. C. Burns and M. P. Glode, "Kawasaki syndrome," The Lancet, vol. 364, no. 9433, pp. 533-544, 2004.

[15] C. L. Wang, Y. T. Wu, C. A. Liu, H. C. Kuo, and K. D. Yang, "Kawasaki disease: infection, immunity and genetics," The Pediatric Infectious Disease Journal, vol. 24, no. 11, pp. 9981004, 2005.

[16] K. Furusho, K. Sato, T. Soeda et al., "High-dose intravenous gammaglobulin for Kawasaki disease," The Lancet, vol. 2, no. 8363, p. 1359, 1983.

[17] D. Chbeir, J. Gaschignard, R. Bonnefoy et al., "Kawasaki disease: abnormal initial echocardiogram is associated with resistance to IV Ig and development of coronary artery lesions," Pediatric Rheumatology Online Journal, vol. 16, no. 1, p. 48, 2018.

[18] E. Villain, J. Kachaner, D. Sidi, G. Blaysat, J. F. Piechaud, and E. Pedroni, "Trial of prevention of coronary aneurysm in Kawasaki's disease using plasma exchange or infusion of 
immunoglobulins," Archives Françaises de Pédiatrie, vol. 44, no. 2, pp. 79-83, 1987.

[19] M. Shinohara, K. Sone, T. Kobayashi, T. Kobayashi, T. Kosuda, and Y. Okada, "Letter | The Journal of Pediatrics - Volume 129, Issue 3," The Journal of Pediatrics, vol. 129, no. 3, pp. 483-484, 1996.

[20] Y. Sun, T. Huang, L. Hammarstrom, and Y. Zhao, "The immunoglobulins: new insights, implications, and applications," Annual Review of Animal Biosciences, vol. 8, pp. 145-169, 2020.

[21] A. H. Rowley and S. T. Shulman, "Pathogenesis and management of Kawasaki disease," Expert Review of Anti-Infective Therapy, vol. 8, no. 2, pp. 197-203, 2010.

[22] JCS Joint Working Group, "Guidelines for diagnosis and management of cardiovascular sequelae in Kawasaki disease (JCS 2013)," Circulation Journal, vol. 78, no. 10, pp. 2521-2562, 2014.

[23] K. Takahashi, T. Oharaseki, and Y. Yokouchi, "Pathogenesis of Kawasaki disease," Clinical and Experimental Immunology, vol. 164, Supplement 1, pp. 20-22, 2011.

[24] H. Muta, M. Ishii, M. Yashiro, R. Uehara, and Y. Nakamura, "Late intravenous immunoglobulin treatment in patients with Kawasaki disease," Pediatrics, vol. 129, no. 2, pp. e291-e297, 2012.

[25] R. Ae, J. Y. Abrams, R. A. Maddox et al., "Outcomes in Kawasaki disease patients with coronary artery abnormalities at admission," American Heart Journal, vol. 225, pp. 120-128, 2020.

[26] A. K. Bal, D. Prasad, P. M. Umali, E. Mammen-Prasad, and A. Petrova, "Timing of intravenous immunoglobulin treatment and risk of coronary artery abnormalities in children with Kawasaki disease," Pediatrics and Neonatology, vol. 55, no. 5, pp. 387-392, 2014.

[27] A. H. Rowley and S. T. Shulman, "Recent advances in the understanding and management of Kawasaki disease," Current Infectious Disease Reports, vol. 12, no. 2, pp. 96-102, 2010.

[28] H. Muta, M. Ishii, K. Egami et al., "Early intravenous gammaglobulin treatment for Kawasaki disease: the nationwide surveys in Japan," The Journal of Pediatrics, vol. 144, no. 4, pp. 496-499, 2004.

[29] S. Wanitkun, "Early intravenous gamma-globulin treatment for Kawasaki disease: the nationwide surveys in Japan," The Journal of Pediatrics, vol. 146, no. 1, pp. 149-150, 2005, 150151.

[30] K. Williams, "Preventing long-term cardiac damage in pediatric patients with Kawasaki disease," Journal of Pediatric Health Care, vol. 31, no. 2, pp. 196-202, 2017.

[31] J. Y. Abrams, E. D. Belay, R. Uehara, R. A. Maddox, L. B. Schonberger, and Y. Nakamura, "Cardiac complications, earlier treatment, and initial disease severity in Kawasaki disease," The Journal of Pediatrics, vol. 188, pp. 64-69, 2017.

[32] K. Y. Lee, J. W. Han, J. S. Lee, and K. T. Whang, "Alteration of biochemical profiles after high-dose intravenous immunoglobulin administration in Kawasaki disease," Acta Paediatrica, vol. 91, no. 2, pp. 164-167, 2002.

[33] T. P. Klassen, P. C. Rowe, and A. Gafni, "Economic evaluation of intravenous immune globulin therapy for Kawasaki syndrome," The Journal of Pediatrics, vol. 122, no. 4, pp. 538542, 1993.

[34] H. Yanagawa, Y. Nakamura, K. Sakata, and M. Yashiro, "Use of intravenous gamma-globulin for Kawasaki disease: effects on cardiac sequelae," Pediatric Cardiology, vol. 18, no. 1, pp. 19-23, 1997.

[35] J. Cui, Y. Zhou, H. Hu, L. Zhao, Z. Du, and H. Du, "PGK1 as an immune target in Kawasaki disease," Clinical and Experimental Immunology, vol. 194, no. 3, pp. 371-379, 2018.

[36] T. Hara, Y. Nakashima, Y. Sakai, H. Nishio, Y. Motomura, and S. Yamasaki, "Kawasaki disease: a matter of innate immunity," Clinical and Experimental Immunology, vol. 186, no. 2, pp. 134-143, 2016.

[37] J. C. Burns, Y. Song, M. Bujold et al., "Immune-monitoring in Kawasaki disease patients treated with infliximab and intravenous immunoglobulin," Clinical and Experimental Immunology, vol. 174, no. 3, pp. 337-344, 2013.

[38] K. Nakatani, S. Takeshita, H. Tsujimoto, Y. Kawamura, H. Kawase, and I. Sekine, "Regulation of the expression of Fc gamma receptor on circulating neutrophils and monocytes in Kawasaki disease," Clinical and Experimental Immunology, vol. 117, no. 2, pp. 418-422, 1999.

[39] J. Hirao, N. Yoshimura, N. Homma, and K. Kano, "Immunological studies on Kawasaki disease: II. Isolation and characterization of an immunosuppressive factor in acute phase sera," Clinical and Experimental Immunology, vol. 67, no. 2, pp. 433-440, 1987.

[40] A. H. Rowley, "The complexities of the diagnosis and management of Kawasaki disease," Infectious Disease Clinics of North America, vol. 29, no. 3, pp. 525-537, 2015.

[41] E. Seo, J. J. Yu, H. O. Jun et al., "Prediction of unresponsiveness to second intravenous immunoglobulin treatment in patients with Kawasaki disease refractory to initial treatment," Korean Journal of Pediatrics, vol. 59, no. 10, pp. 408-413, 2016.

[42] M. Yoshida, S. Oana, H. Masuda et al., "Recurrence of fever after initial intravenous immunoglobulin treatment in children with Kawasaki disease," Clinical Pediatrics (Phila), vol. 57, no. 2, pp. 189-192, 2018.

[43] M. S. Lo and J. W. Newburger, "Role of intravenous immunoglobulin in the treatment of Kawasaki disease," International Journal of Rheumatic Diseases, vol. 21, no. 1, pp. 64-69, 2018.

[44] S. C. Roberts, S. Jain, A. H. Tremoulet et al., "The Kawasaki disease comparative effectiveness (KIDCARE) trial: a phase III, randomized trial of second intravenous immunoglobulin versus infliximab for resistant Kawasaki disease," Contemporary Clinical Trials, vol. 79, pp. 98-103, 2019. 